\title{
Agroecologia e promoção da saúde no Brasil
}

\author{
Elaine de Azevedo ${ }^{1}$ e Maria Cecília Focesi Pelicioni ${ }^{1}$
}

Como citar Azevedo E, Pelicioni MCF. Agroecologia e promoção da saúde no Brasil. Rev Panam Salud Publica. 2012;31(4):290-5.

RESUMO Objetivo. Pesquisar como os especialistas da promoção de saúde e da agroecologia compreendem os conceitos dessas áreas de diretrizes comuns e como é concebida a relação entre tais conceitos.

Métodos. Pesquisa qualitativa. Foram realizadas entrevistas com 14 especialistas das duas áreas sobre relações entre sistema agroalimentar e saúde; conceitos de agroecologia e promoção da saúde; relevância da inserção da agroecologia nos cursos de formação de saúde pública e vice-versa.

Resultados. Existe pouco diálogo entre os campos de estudo que foram considerados afins, sendo a qualidade do alimento a principal interface entre as áreas. A agroecologia apareceu como um sistema de produção de alimentos saudáveis, mas o estudo mostrou outras relações: agroecologia e empowerment, fomento à autonomia e qualidade de vida e melhores condições socioeconômicas para o agricultor; agroecologia e saúde ambiental; agroecologia e participação social; agroecologia, territorialidade e resgate cultural; agroecologia, alimentos locais e baixo custo produtivo. Já a promoção de saúde foi essencialmente relacionada a práticas voltadas à manutenção de estilos de vida saudável. Os especialistas mostraram-se favoráveis à inserção de conhecimentos da área da saúde pública na agroecologia e vice-versa.

Conclusões. A agroecologia e a promoção da saúde são áreas contributivas e complementares, cuja aproximação pode vir a enriquecer a discussão da saúde rural e a concepção das politicas públicas que se debruçam sobre essa temática, estimulando intervenções e práticas intersetoriais.

Palavras-chave Agricultura sustentável; promoção da saúde; saúde da população rural; Brasil.

Estudos sobre promoção da saúde surgiram no Canadá, na década de 1970, orientados por uma visão de saúde que considera as diversas causas do binômio saúde-doença, a partir de diretrizes éticas de fomento à democracia e à equidade, de estímulo à participação popular e de promoção da sustentabilidade e da qualidade de vida dos indivíduos (1-2). O ideário de promoção da saúde trabalha com a ideia de responsabilização múltipla, seja pelos problemas, ou soluções propostas para os mesmos, combinando ações intersetoriais (3).

\footnotetext{
Universidade Federal da Grande Dourados, Faculdade de Ciências da Saúde, Dourados (MS), Brasil. Enviar correspondência a: Elaine de Azevedo, elainepeled@gmail.com
}

A agroecologia apareceu como disciplina científica na década de 1930. A partir de 1960, o ideário se mesclou com o movimento ambientalista questionando o sistema agroalimentar moderno. Como prática agrícola, estabeleceu-se nos anos 1980 (4). Destacou-se de outras formas de agricultura sustentável ao assumir o caráter polissêmico de movimento econômico, ético e sociopolítico que objetiva centralmente fortalecer a identidade do agricultor familiar, resgatando suas raízes culturais e sua autonomia (4-5). Para isso, esse agricultor foi estimulado a se inserir em uma estrutura social agrária associativa e cooperada, baseada em práticas tradicionais e locais que o mantenha independente do complexo industrial agrícola externo a sua propriedade (6). Como consequência disso, a forma de produzir alimentos na perspectiva agroecológica tem baixo impacto ambiental e promove a qualidade de vida. Assim, a implantação de um sistema produtivo sustentável nos âmbitos social, ambiental e econômico passou a ser, igualmente, seu objetivo (7).

Apesar da importância da agricultura familiar que produz $80 \%$ da alimentação no Brasil (8) e dos desafiantes objetivos da agroecologia, a saúde rural sob a ótica da agricultura sustentável tem sido pouco explorada em pesquisas da saúde pública e coletiva. Um sistema que minimize as repercussões ambientais negativas dessa atividade e que considere 
o bem-estar do agricultor pode ampliar o conceito de promoção de saúde nesse território, com significativas repercussões no meio urbano.

Além de objetivos comuns, existe outro fator de aproximação desses ideários. Fora do âmbito dos especialistas, a percepção sobre os mesmos tende a ser estreita e reducionista. A agroecologia tem sido simplificadamente relacionada a um sistema produtivo que não utiliza agrotóxicos, sendo pouco conhecidas suas dimensões política e socioambiental. Já a promoção da saúde é recorrentemente percebida como um conjunto de práticas individuais e restritivas, relacionadas a estilos de vida saudáveis, desqualificando a discussão acerca das condições de vida como determinantes da saúde.

Diante das escassas relações conceituais entre esses dois campos de interfaces comuns e complementares, julgou-se relevante apresentar esse estudo, de caráter qualitativo, que analisou a opinião de especialistas das duas áreas para saber como compreendem os conceitos de agroecologia e promoção da saúde e como concebem as relações entre ambos.

\section{MÉTODOS}

A técnica utilizada em campo nesta pesquisa qualitativa foi a entrevista semi-estruturada, entendida como um processo de interação social por meio do qual o pesquisador buscou informações a partir de roteiro que abordava a problemática central (9).

Os informantes expressaram livremente suas percepções sobre as relações entre o sistema agroalimentar e a saúde; sobre agroecologia (no caso dos especialistas em saúde pública) e promoção da saúde (para os especialistas em agroecologia); e, por fim, deram opiniões sobre a relevância da inserção da agroecologia nos cursos de formação em saúde pública e coletiva e vice-versa.

O critério de seleção dos 14 entrevistados foi baseado na atuação de cada um como docente em universidade públicas em cursos de agroecologia e saúde pública e coletiva. Foram entrevistados professores de agroecologia das Universidades Federais de Santa Catarina e de São Carlos e da Universidade de São Paulo e, na área de saúde pública, docentes da Universidade de São Paulo, da Universidade Federal do ABC e da Universidade Federal de São Paulo.
Iniciou-se com dois professores titulares, referências de cada área, dos dois primeiros cursos de nível superior em agroecologia e saúde pública no Estado de São Paulo, na Universidade Federal de São Carlos e na Universidade de São Paulo, respectivamente. Com a técnica conhecida como Bola de Neve (10), os participantes indicaram os próximos entrevistados, até o ponto de saturação das informações coletadas.

Após a apresentação dos propósitos da pesquisa e da assinatura do Termo de Consentimento, as entrevistas com duração aproximada de 45 minutos foram conduzidas pela primeira autora do estudo. Foram gravadas e transcritas e seu conteúdo foi utilizado integralmente.

O material foi explorado na busca de padrões recorrentes e discordantes, temáticas salientes e relevantes para os entrevistados, expressões idiomáticas, metáforas e elementos norteadores. Após a análise, alguns códigos foram elaborados de forma indutiva para identificar temas emergentes (11).

Como resultado desse processo reflexivo, intercalado a consultas a autores de referência sobre o tema da pesquisa, foi possível construir e definir categorias analíticas para o trabalho, apresentadas no item Resultados.

A pesquisa atendeu a Resolução 196/96 e foi aprovada pelo Comitê de Ética da Faculdade de Saúde Pública da Universidade de São Paulo, em 2010. Para preservar a identidade dos especialistas, suas falas literais aparecerão entre aspas ou em recuo e o autor das mesmas será aqui mantido no gênero masculino, mesmo tendo havido diversificação de gênero entre os informantes.

\section{RESULTADOS}

O estudo mostrou que o tempo de graduação dos entrevistados variou entre 20 a 40 anos. Todos possuíam, no mínimo, o grau de doutor. Os especialistas da agroecologia eram todos agrônomos e fizeram pós-graduação em áreas variadas (desenvolvimento rural, meio ambiente, sociologia rural, desenvolvimento sustentável, etologia, genética e biodiversidade e agroecologia). Já os especialistas da promoção da saúde, apresentaram diversidade nas suas graduações (enfermagem, pedagogia, ciências sociais, biologia, medicina) e possuíam uma pós-graduação em comum: saúde pública. Todos se dedicaram a pesqui- sas nos últimos cinco anos sobre temas ligados a agroecologia ou a promoção da saúde em diferentes espaços: comunidades, escolas, associações, serviços de saúde, entre outros.

\section{Relação entre sistema agroalimentar e saúde}

De forma geral, os entrevistados concordaram que a relação entre sistema agroalimentar e saúde procede e o primeiro foi mencionado como um "irmão da saúde pública". Entretanto, essa relação não se estabeleceu de imediato para todos e, algumas vezes, surgiu uma visão negativa: a agricultura como uma atividade de risco ambiental e social; a questão do êxodo rural - " "a retirada do espaço conhecido do agricultor, do seu fazer, do seu viver" - vinculou o padrão produtivo a processos de instabilidade social; e o alimento tradicionalmente percebido como elemento promotor de saúde foi relacionado a doenças. "O sistema agroalimentar é, na verdade, promotor de doença, especialmente pelo uso exagerado de agrotóxicos".

Uma temática emergente foi a qualidade dos alimentos — "saúde passa muito pelo alimento" - ressaltando a necessidade de considerar sua origem e a isenção de agrotóxicos. Surgiu também a noção de qualidade da dieta relacionada a restrição de gorduras, açúcar, sal e calorias.

Além do tema da qualidade e inocuidade química dos alimentos, foi levantada a preocupação com a saúde ocupacional dos agricultores. Uma das falas reforçou a discussão que envolve a multifuncionalidade da agricultura (12) e os seus custos socioambientais: "a função da agricultura não é produzir a todo custo; além da renda do agricultor, ela tem uma série de outras tarefas e isso merece ser debatido com maior atenção nas políticas públicas".

Foi observada uma perspectiva plural de qualidade de vida e saúde a partir de formas de agricultura sustentável. Alguns dos entrevistados incluíram a agroecologia nessa relação e destacaram aspectos importantes como: a agroecologia como instrumento de empowerment e fomento à autonomia dos agricultores para "tomar em suas mãos o processo produtivo"; a saúde do agricultor relacionada ao "tipo de tecnologia que ele usa"; a possibilidade de maiores "lucros para o agricultor agroecológico" 
e de lazer no meio rural "através do agroturismo"; agroecologia e promoção da segurança alimentar, saúde e conservação ambiental. "Uma agroecologia pra promoção da saúde" enfatizou um informante.

Tais aspectos são sempre mencionados por autores que se dedicam à agroecologia (4-7). Entretanto, esses elementos não são apresentados como determinantes de saúde, como elabora esse entrevistado sobre a relação entre agricultura e saúde ambiental:

(...) uma agricultura menos poluidora é uma agricultura que faz bem pro meio ambiente, pra saúde do planeta e, portanto, pra saúde humana. Prá cada um de nós enquanto indivíduo (. . .) não dá pra pensar na saúde do indivíduo separada da saúde ambiental e saúde do planeta (. . .). Biodiversidade, um ar respirável, tudo isso faz parte da saúde e a agricultura tem sua missão a cumprir nessa ideia de que a saúde do planeta deva melhorar.

O modo como as pessoas ou grupos sociais vivenciam a saúde está intimamente relacionado ao contexto cultural e à organização de cada grupo; o elemento cultural salienta o fato de que a saúde não se reduz a uma evidência orgânica, natural e objetiva (13). Esse elemento foi destacado como um determinante da saúde do agricultor, inserindo a temática em uma dimensão mais subjetiva:

Penso na cultura, nas relações e nos problemas das pessoas que se dedicam à agricultura. Isso é importante pra promoção da saúde. A atividade econômica é importante, mas ela marca todo um padrão de vida cultural e de saúde dessa população.

Nessa mesma dimensão, o estreitamento da relação entre sistema agroalimentar e saúde foi percebido como um "fator de promoção de redes sociais e de aglutinação", estimulando a participação social.

A questão da proximidade entre a produção e o consumo de alimentos como aspecto salutar apareceu em uma citação que priorizava o consumo de alimentos "que cheguem o mais livre de intermediários possível". Tal citação leva ao conceito de alimento local produzido com menos gasto de energia e ajustado ao bioma da região de produção e de consumo.

A relação estabelecida entre agricultura e saúde foi sempre a partir de formas de agricultura sustentável, dentro da qual "o indivíduo produz a sua forma de viver".

\section{Visão da agroecologia para os especialistas em promoção da saúde}

Grande parte das respostas constituíram inicialmente uma afirmação de pouco conhecimento sobre a agroecologia e muitos se consideravam "leigos no assunto". Entretanto, na continuidade das entrevistas construíram-se relações complexas, seguindo a mesma tendência das opiniões supracitadas.

A resposta mais comum baseou-se no prefixo 'agro' do termo agroecologia, uma forma de agricultura de baixo impacto ambiental que prescinde de agrotóxicos: "o que vem na cabeça é o uso de formas de cultivo que deveriam ser menos agressivas ao meio ambiente. Possivelmente usar melhor a terra, em vez de defensivos, usar composteira".

Outras respostas endossaram a relação da agroecologia com a saúde, destacando "que a forma de usar a terra tem a ver com a saúde das pessoas", do produtor ao consumidor:

A agroecologia seria a produção de alimentos de uma forma que produzisse uma boa qualidade de vida no final da cadeia, qualidade de vida para as duas pontas; para o agricultor evitando o uso de agrotóxicos e contaminação e para o consumidor, da mesma forma, produzindo um alimento saudável.

Um dos informantes com pouco conhecimento sobre a temática julgou que os cursos de formação em agroecologia já estavam inseridos na área da saúde, pois "não tem como a saúde estar fora disso".

Reapareceram abordagens socioeconômicas de um sistema que se "opõe ao agronegócio de exportação". A agroecologia foi novamente apresentada como uma possibilidade de fortalecimento do agricultor familiar, como uma estratégia para mantê-lo no meio rural com dignidade e como prática de conservação ambiental, minimizando gastos com a produção e o transporte de alimentos. Ressurgiram também os aspectos mais subjetivos, como agroecologia, resgate do sentimento de territorialidade e manutenção de povos e saberes tradicionais: “(. . . ) valorizar o saber popular, as pessoas, e isso é promotor". Destacou-se também a questão do sabor do alimento produzido "que deve ser diferente, mais gostoso".

\section{Visão da promoção da saúde para os especialistas em agroecologia}

Ninguém parecia ter dúvidas sobre o conceito de promoção da saúde, mas grande parte tratou do termo em si, e não do campo de estudos.

A maioria vinculou promoção de saúde a práticas voltadas à manutenção de estilos de vida saudável, abordagem que tende a desconsiderar as condições de vida e os determinantes sociais da saúde: "eu me lembro das pessoas fazendo ginástica na rua, a terceira idade correndo na beira-mar, sei lá, mais ou menos isso (. . .) eu acho que a melhor maneira de promover saúde é uma alimentação limpa e balanceada e esporte". "Os meios de comunicação tratam muito de noções do tipo: é preciso comer mais hortaliças, mais frutas, fazer caminhadas .. . Usar menos álcool, comer menos açúcar, gorduras, cuidar do tabagismo".

Outras perspectivas surgiram como: "promoção da saúde? "ah, trabalho com periferia, orientação com nutrição, esse tipo de coisa". A alimentação e a contaminação química apareceram também em duas falas: "a gente devia se preocupar com a saúde pelo envenenamento por compostos químicos desconhecidos; o grande problema é o agrotóxico". "Uma política pública de promoção da saúde pensa no alimento. Fiscaliza como está sendo produzido, como chega na mesa do consumidor". Esse mesmo entrevistado relacionou a promoção a ações preventivistas da saúde pública como vacinação e controle de medicamentos "de qualidade, seguros e de livre acesso às pessoas".

A promoção de saúde como processo vinculado à responsabilidade do indivíduo frente a sua saúde surgiu na percepção de um entrevistado:

Se as pessoas tiverem uma maior responsabilização na própria saúde, isso seria muito eficaz em termos de promoção da saúde, ou seja, as políticas 
de promoção da saúde poderiam ser nesse sentido, o de favorecer uma responsabilização maior com sua própria saúde e com a saúde do planeta e isso levaria a uma perspectiva mais de prevenção. Cada um deveria ter meios para se responsabilizar e tomar a saúde nas próprias mãos.

\section{Relevância da promoção da saúde para a formação profissional em agroecologia e da agroecologia para a formação profissional em saúde pública}

Diante dessa questão, muitos dos entrevistados assumiram uma postura reflexiva: "eu nunca tinha pensado muito nisso". Entretanto, a maioria afirmou que a relação entre os campos de estudo é pertinente, "como um tema transversal na Saúde Pública", ou ainda, "uma interface que não tem sido explorada devidamente", "(. . .) uma discussão muito rejeitada, pois a Agronomia é um ambiente meio polarizado", segundo um especialista da Agroecologia.

Foi mencionado o desafio da universidade de "responder às necessidades sociais" que se apresentam, bem como a dificuldade dos alunos de graduação em compreender a complexidade dos determinantes sociais do processo saúdedoença; assim sendo, a agroecologia não "devia ser inserida tão cedo nos currículos de cursos de graduação da área médica". Para esse entrevistado a pósgraduação acolheria com mais propriedade a discussão "não necessariamente sobre saúde e agroecologia, mas saúde e ambiente; saúde e desenvolvimento local; saúde e outras opções econômicas; saúde e território".

Ressaltou-se a dificuldade de inserir tais relações em cursos da área de saúde que abordam saúde como simples ausência de doença. Para construir esse diálogo interdisciplinar, o informante enfatizou a necessidade de conceber o conceito de saúde na perspectiva da Promoção da Saúde.

Um especialista afirmou que na Saúde Pública, a subárea de Saúde Ambiental poderia absorver a discussão da Agroecologia, incluindo nela os determinantes socioambientais da saúde. Outra ideia foi a de inserir esse conteúdo na graduação de Nutrição, pois "há um desconhecimento sobre o sistema que produz alimento e esse tipo de incorporação discute como se produz o alimento". Um mencionou que nos cursos de Saúde Pública, o conteúdo de Agroecologia "cabe com certeza". E continuou: "(. . .) mas eu não consigo me imaginar dando aulas pra agrônomos, pois eu não sei outros elementos da agronomia".

Foi mais uma vez destacada a concepção do meio rural "como espaço de saúde" se vinculado a práticas agroecológicas.

\section{DISCUSSÃO}

Pelos elementos identificados nessa pesquisa, percebe-se a falta de diálogo entre a Agroecologia e a Promoção da Saúde, consideradas áreas afins, sendo a qualidade do alimento o principal elemento de conexão entre elas. Além da origem dos alimentos e da ausência de agrotóxicos como fator de qualidade, também foi apresentado o conceito de alimento saudável com base em abordagens restritivas, tendência das estratégias de promoção da saúde voltadas às práticas alimentares saudáveis. Porém, nenhum outro contaminante alimentar (fertilizantes, aditivos sintéticos, antibióticos e outras drogas veterinárias) foi mencionado, demonstrando que a produção sustentável é essencialmente associada a alimentos vegetais in natura e à ausência de agrotóxicos.

A definição mais comum da agroecologia foi uma forma de agricultura sustentável, mas o estudo mostrou uma amplitude nas relações estabelecidas pelos especialistas da área da promoção da saúde. Tal fato mostra a familiaridade desses especialistas com diálogos interdisciplinares, talvez por se originarem de diferentes áreas. Percebe-se igualmente o conhecimento das repercussões ambientais, sociais e sobre a saúde humana $(14,15)$ do sistema agroalimentar dominante e da perspectiva plural da agroecologia fora do seu próprio meio especialista.

A agroecologia como prática que estimula a participação social definida como "partilha de processos decisórios e a superação de conflitos de interesse por meio da negociação" (16) parece essencial para apoiar indivíduos socialmente saudáveis e para politizar as áreas da saúde e de produção de alimentos. Essa é uma das diretrizes da Política Nacional de Promoção da Saúde que objetiva: "fortalecer a participação social como fundamental na consecução de resultados de promoção da saúde, em especial a equidade e o empowerment individual e comunitário" (17).

O destaque dado ao conceito de alimento local é relevante como questão ambiental, uma vez que sete a dez calorias de combustível fóssil são usadas para produzir uma caloria de energia alimentícia; $1 / 5$ dessas calorias vai para produção e o restante é utilizado no beneficiamento e transporte desse alimento (18). Além disso, sob a ótica da agroecologia a produção de alimentos locais contribui para viabilizar circuitos regionais de comercialização e para estimular relações mais horizontais de produção e consumo via comércio varejista de pequeno porte, construindo mecanismos que permitem a cooperação, a reciprocidade e o fomento de ações coletivas (19).

O conceito de promoção da saúde entre os especialistas agroecológicos foi mais estreito. Sendo um grupo mais homogêneo, sintonizado com as premissas interdisciplinares da sua própria especialidade, não tem muitas referências das duas tendências conceituais presentes nos estudos de promoção da saúde; a primeira, centrada na responsabilização do indivíduo a partir do seu comportamento individual e estilo de vida. A outra considera os determinantes sociais da doença e o desenvolvimento de políticas públicas e condições favoráveis à saúde (20).

Esse desconhecimento é compreensível, uma vez que até o início de 1990 quando ocorreram os primeiro eventos internacionais sobre essa temática no hemisfério sul, as ações da promoção da saúde respondiam as necessidades da população dos países mais desenvolvidos economicamente, com intervenções ajustadas a sua realidade. Além disso, muitas das ações da Política Nacional de Promoção da Saúde do Brasil homologada em 2006 seguem essa direção, o que gera uma crítica recorrente a tal política.

Para Verdi e Caponi (21), é o reducionismo que explica a adoção de estratégias de informação e incentivo a mudanças de estilos de vida individuais, mantendo o antigo enfoque preventivista na área da saúde. Segundo essas autoras, o olhar para os desvios de conduta individuais deslocam o cerne da questão do corpo social para o corpo biológico ou físico, bem como a responsabilidade do Estado 
na produção de respostas efetivas para o próprio indivíduo. Ao assumir tal tendência, é preciso evitar a armadilha de culpabilização das próprias vítimas e deixar de fortalecer a agenda que indica os investimentos em saúde e a redução das desigualdades sociais e seus fatores determinantes como os segmentos com maior impacto de ganho para o estado de saúde, qualidade de vida e bem-estar da população.

Os dois grupos consideraram pertinente a aproximação dos campos de estudos e sua inserção nos cursos de formação em saúde pública/coletiva e em agroecologia. Porém, apareceram dúvidas de como isso seria possível, revelando a dificuldade da implementação de propostas interdisciplinares, especialmente em cursos de graduação. A prática interdisciplinar não exige o conhecimento específico de outras áreas, mas pressupõe a busca de interfaces comuns entre as diferentes áreas e a coexistência entre diversos saberes e especialistas.

Inserir a agroecologia nos cursos da área da saúde sob as premissas da promoção da saúde pode se tornar uma estratégia de superação da hegemonia do modelo biomédico intervencionista ainda prevalente. Na agronomia, essa inserção poderá conscientizar o agrônomo do seu papel de promotor da saúde em diferentes níveis.

$\mathrm{O}$ estudo parece reforçar a premissa de que para discutir saúde no meio rural é essencial mobilizar conceitos que envolvam as diferentes formas de agricultura sustentável.

Essa é uma temática pouco debatida que necessita de aprofundamento e de novas pesquisas que se sobreponham aos fatores limitantes deste estudo que foram a impossibilidade de abordar um universo maior de especialistas, incluindo outras universidades e outros
Estados; a necessidade de realizar estudos com profissionais da saúde pública e da agronomia em regiões e espaços onde tais conhecimentos não são explorados, sensibilizando novos atores e buscando outros questionamentos.

Por fim, pode-se afirmar que a agroecologia e a promoção da saúde são áreas contributivas e complementares, cuja aproximação pode vir a enriquecer a discussão em torno da saúde rural e a concepção das políticas públicas que se debruçem sobre elas, estimulando intervenções e novas práticas intersetoriais.

Agradecimentos. Elaine Azevedo agradece o apoio da Fundação de Amparo à Pesquisa do Estado de São Paulo (FAPESP 09/54418-0) durante a realização desta pesquisa no período de pós- doutorado na Faculdade de Saúde Pública da Universidade de São Paulo, em 2010.

\section{REFERÊNCIAS}

1. Pelicioni MCF. Promoção da saúde e meio ambiente: uma trajetória técnica-política. In: Philippi Jr A, Pelicioni MCF, eds. Educação ambiental e sustentabilidade. $1^{\circ}$ Edição. São Paulo - SP: Manole; 2005;413-20.

2. Westphal MF. Promoção da saúde e prevenção de doenças. In: Campos GWS, Minayo MCS, Akerman M, Drumond Junior M, Carvalho YM (orgs). Tratado de Saúde Coletiva. $1^{\text {a }}$ Edição. Rio de Janeiro - RJ: Editora Fiocruz/São Paulo - SP: Hucitec; 2006;635-67.

3. Buss PM. Promoção da saúde e qualidade de vida. Cienc Saude Coletiva. 2000;5(1):16378.

4. Wezel A, Bellon S, Doré T, Francis C, Vallod D, David C. Agroecology as a science, a movement and a practice. A review. Agron Sustain Dev. 2009;29(4):503-15.

5. Caporal FR (org), Costabeber JA, Paulus G. Agroecologia: uma ciência do campo da complexidade. $1^{a}$ Edição. Brasília - DF: MDS/ Embrapa; 2009.

6. Assis RL, Romeiro AR. Agroecologia e agricultura familiar na região centro-sul do estado do Paraná. Rev Econ Sociol Rural. 2005;43(1):155-77.

7. Gliessman SR. Agroecologia: Processos ecológicos em agricultura sustentável. $2^{\circ}$. Edição. Porto Alegre - RS: UFRGS; 2001.

8. Instituto Brasileiro de Geografia e Estatística. Censo Agropecuário 2006. Rio de Janeiro: IBGE; 2006. Disponível em: http://www. ibge.gov.br/home/estatistica/economia/ agropecuaria/censoagro/2006/agropecu ario.pdf Acessado em agosto de 2010.

9. Haguette TMF. Metodologias qualitativas na Sociologia. $6^{\circ}$ Edição. Petrópolis - RJ: Vozes; 1999.

10. Salganik MJ, Heckathorn DD. Sampling and Estimation in Hidden Populations using Respondent-Driven Sampling. Sociological Methodology. 2004;34(1):193-239.

11. Minayo MCS. O desafio do conhecimento: Pesquisa Qualitativa em Saúde. $10^{\circ}$ Edição. São Paulo — SP: Hucitec/Rio de Janeiro - RJ: Abrasco; 2007.

12. Maluf RS, Bonnal P, Cazella AA. Multifunctionality of agriculture and territorial development: implications and challenges in combining the approaches. Estud Soc Agric [online] 2008;4, Selected Edition.

13. Minayo MCS. Contribuições da Antropologia para pensar e fazer saúde. In: Campos GWS, Minayo MCS, Akerman M, Drumond Junior M, Carvalho YM (orgs). Tratado de Saúde Coletiva. Rio de Janeiro - RJ: Editora Fiocruz/ São Paulo — SP: Hucitec; 2006;189-210.

14. Bruinsma J. World agriculture: towards 2015/2030: an FAO perspective. Rome: FAO. London: Earthscan; 2003.

15. Azevedo E. Alimentos Orgânicos: ampliando conceitos de saúde humana, social e ambiental. $2^{\circ}$ Edição. Tubarão - SC: Editora Unisul; 2006.
16. Bógus CM, Westphal MF. Participação social e cidadania em movimentos por cidades saudáveis. In: Fernandez JCA, Mendes R (orgs). Promoção da Saúde e Gestão local. São Paulo - SP: Aderaldo \& Rothschild (Hucitec); 2007;61-83.

17. Política Nacional de Promoção da Saúde. Brasília: Ministério da Saúde. 2006. Disponível em: http://portal.saude.gov.br/portal/ arquivos/pdf/PNPS2.pdf Acessado em 6 de dezembro de 2010.

18. Pollan M. O Dilema do Onívoro: uma história natural de quatro refeições. $1^{\circ}$ Edição. Rio de Janeiro — RJ: Intrínseca; 2007.

19. Altieri M. Agroecologia: bases científicas para uma agricultura sustentável. $1^{\circ}$ Edição. Guaíba - RS: Ed. Agropecuária; 2002.

20. Buss PM. Uma introdução ao conceito de promoção da saúde. In: Czeresnia D, Freitas CM (orgs). Promoção da saúde: conceitos, reflexões, tendências. $3^{\circ}$ Edição. $\mathrm{O}$. Rio de Janeiro - RJ: Fiocruz; 2003;15-38.

21. Verdi M, Caponi S. Reflexões sobre a promoção da saúde numa perspectiva bioética. Texto Contexto Enferm. 2005;14(1):82-8.

Manuscrito recebido em 18 de fevereiro de 2011. Aceito em versão revisada em 28 de setembro de 2011. 
ABSTRACT Objective. Research how specialists in health promotion and agroecology understand the concepts in those areas of common guidelines and how the relationship between such concepts is conceived.

\section{Agroecology and health}

Methods. Qualitative research. Fourteen specialists in the two areas were interviewed about the relationship between the agrofood system and health, concepts of agroecology and health promotion, and the relevance of including agroecology in public health training courses and vice-versa.

Results. There is little dialogue between the fields of study that were considered similar, food quality being the main interface between the areas. agroecology appeared to be a system of healthy food production, but the study showed other connections: agroecology and empowerment, a spur to autonomy and quality of life, and better socioeconomic conditions for the farmer; agroecology and environmental health; agroecology and community involvement; agroecology, territoriality, and cultural rescue [translator's note: this is a term for measures taken to revitalize or preserve imperiled indigenous cultures]; and agroecology, local foods, and low costs of production. Health promotion already was linked in effect to practices oriented to healthy lifestyles. The specialists appeared favorable toward including knowledge about public health in agroecology and vice-versa.

Conclusions. Agroecology and health promotion contribute to one another and are complementary, and bringing them closer together can lead to an enriched discussion about rural health and the concept of public policies that focus on this theme, thereby stimulating actions for improvement and intersectoral practices.

Key words Sustainable agriculture; health promotion; rural health; Brazil. 